\title{
Some celebratory HCI reflections on a celebratory HCI festschrift
}

\author{
John Long
}

University College London, UCL Interaction Centre, MPEB 8th Floor, Gower Street, London WC1E 6BT, United Kingdom

'And so I face the final curtain, Regrets I’ve had a few' (Sinatra).

but on celebratory reflection

'Non, rien de rien,

Non, je ne regrette rien' (Piaf).

Festschrifts are meant to be celebratory (Wikipedia, 2009). My reflections too. First, I celebrate the very idea of an HCI festschrift. A bit premature perhaps; but better early, than too late, and it will serve to encourage others. Congratulations, then, to Alistair and Ann, along with Diane, the IWC publishers and the festschrift authors for making it happen. I am very touched. However, this festschrift cannot be of the traditional kind. HCI is still in its early stages. Trends and visions continue to come and go. The field is too immature for any consensus agreement to celebrate individual contributor's legacies to HCI discipline progress. In this respect, it does not help, that my own work has been intimately bound up for 30 years with that of colleagues. First, from the MRC Applied Psychology Unit, Cambridge (MRC/APU), then from the Ergonomics and HCI Unit at University College London (EU/UCL). Even reflecting on one's own festschrift might be considered unusual by some (again, all credit to the editors).

Second, I would like to celebrate myself. After all, a festschrift needs someone to honour.

However, having reflected long and hard about how I got here, I have not come up with much by way of an explanation, other than having the luck to work with bright and engaging colleagues. As an aside, I put down what success I have enjoyed to never working with $\mathrm{PhD}$ students not cleverer than myself and never working with MSc students cleverer than myself. When I came across the latter, I converted them into the former (they know who they are).

Third, I would like to celebrate the world of HCI. Obviously, students, practitioners and researchers, who identify themselves with $\mathrm{HCI}$ and who together make up the HCI community. But also IT professionals, outside the community, who do not identify with HCI; but who actually 
design so many of the interfaces in use today. Most IT interfaces continue to be designed and implemented by such professionals. We forget them at our (professional) peril (see my 'Hopes' for HCI later).

Fourth, I would like to celebrate the attempts of the HCI community to make of itself an HCI design discipline (or disciplines). Twenty years ago, craft, applied science and engineering were identified as: 'possible alternative and equally legitimate' such attempts' (Long and Dowell, 1989, in their Discipline Conception for HCI). These attempts (and others) continue and should be celebrated, because, as they point out, one discipline 'might be usefully but indirectly informed by the discipline knowledge of another'. Further, such mutual support 'maximises the exploitation of what is known and practised in HCI. . . it encourages the notion of a community of HCI, superordinate to that of any single discipline...' (also from Long and Dowell, whose greater truth is conceded by Carroll (2010)).

Fifth, I would like to celebrate HCI engineering, as one of these attempts to make of HCI a design discipline. Dowell and Long $(1989,1998)$ argue that such a discipline would acquire design knowledge in the form of design principles. The latter would support the diagnosis of design problems (see Hill, 2010) and the prescription of design solutions (see Salter, 2010). The design practice would be 'specify then implement' (the principle ensuring that the design solution required no testing). The scope of such principles would be determined by the 'hardness' of the design problems (that is, the extent to which they can be specified) and the (relative) determinism of the human behaviours, which the principles implicate (within the limits required for a particular design solution, for example, as in road traffic system protocols). Dowell and Long propose a conception for the general HCI design problem (referred to here as the HCI Design Problem Conception) of 'users interacting with computers to perform work effectively'. The Conception 'expresses the problem more formally and which might be embodied in (HCI) engineering principles'.

Sixth, I would like to celebrate the members and the work (referred to here as EU research) of the EU/UCL. Members included: MSc and PhD students; academic and administrative staff; visitors; and researchers. It was the greatest place to work and play (both hard). Early work consisted of psychology, writing up research from my MRC/APU, Cambridge PhD days (Long, 1980); applied psychology, originating with Donald Broadbent, my PhD supervisor (Long, 1995) and now under the new guise of Cognitive Ergonomics (Long and Whitefield, 1989). However, all of the EU research would consider itself to be engineering, of one sort or another. It attempted to advance the state of HCI in the short to medium term. All used the Discipline Conception. Most used the Design Problem Conception. The work covered most areas of HCI: user requirements (a method for multi-disciplinary practice - Denley and Long, 2001); design (MUSE a Method for Usability Engineering - Lim and Long, 1994); and evaluation (a planning aid to support evaluation 
practice - Denley and Long, 1997). Design-oriented substantive knowledge, in the form of user, interactive worksystem and domain models, as well as the methodological knowledge, required for their application to design, were both acquired (Smith, et al., 1997, in the domain of secretarial office administration; Hill and Long, 1996, in the domain of emergency services management; and Timmer and Long, 2002, in the domain of air traffic management - see also Hill, 2010). Later EU research, using both Discipline and Design Problem Conceptions, attempted to acquire formal HCI design principles, as envisaged by Long and Dowell earlier principles, which offer a better guarantee in solving design problems, than other forms of knowledge, such as heuristics, guidelines, models and methods. Better because principles support the derivation of design solutions, given design problems. In the interests of clarity, this research is referred to here as HCI (Principles) Engineering (see Carroll, Wild and Hill later). Early and initial HCI design principles have been proposed for 'hard' problems in the domains of domestic energy management (Stork, 1999) and of business-to-customer electronic commerce (Cummaford, 2007).

Taken together, the two lines of EU research provided support for (iterative) 'specify and implement' design practice (models and methods) and 'specify then implement' practice (principles). Judgement on their success or failure, I leave to others.

Seventh, I would like to celebrate the festschrift papers themselves (both accepted and rejected), their authors, and their reviewers. My natural instinct is to peer review the papers. Space and my honoured status forbid such a review. However, I hope to do this elsewhere (I owe it to the authors and to myself). Instead, in the spirit of the festschrift, I will attempt to address, by way of clarifications, issues problematic for EU research. For copies of papers, referencing the author's work - see Long (2010), this issue.

Carroll's paper (2010) raises some serious issues for the research, as well as offering some (unwitting) complements. Although an unreconstructed 'existentialist' in my private life, I am delighted to be considered a 'positivist' (although perhaps not a 'hoary' one), when it comes to HCI engineering. Far from being discourteous, I take it as a complement. Like one of Carroll's reviewers, any time I fly, I am thankful for the odd positivist engineer in the design team of the airplane. Also a complement to be linked with the name of Alan Newell (in the same sentence, no less). Pity Carroll castigates us both for 'going too far'. Otherwise, Carroll finds little to celebrate in the Long and Dowell Discipline Conception (1989). His celebration is positively (and prematurely) funereal. It is also a pity he focusses only on the Discipline Conception paper, because the issues he raises are all addressed elsewhere. They relate to his doubts concerning: the specifiability of designs; the determinism of human behaviours, implicated by them; and the extended scope of HCI since 1989. 
The issues are not really a problem for EU 'models and methods' research (see earlier). For example, Hill (2010) uses both Discipline and Design Problem Conceptions to diagnose design problems and to reason about design solutions to those problems in the domain of emergency services management. Both are specified well enough to establish the relations between them for the purposes of the research. The human behaviours implicated are those of trained emergency personnel, as specified by co-ordination protocols and so, deterministic enough for the purposes of specifying a possible design solution.

The issues are, however, serious for HCI (Principles) Engineering. They are addressed in Dowell and Long $(1989,1998)$. The specifiability of designs and the determinism of human behaviours, implicated by them, all depend on the 'hardness' of the design problem (see in particular Fig. 2, a classification of design disciplines, which plots discipline practices against discipline knowledge with respect to the 'hardness' or 'softness' of general design problems). Early and initial design principles have already been referenced, concerning EU research (Stork, 1999; Cummaford, 2007). The completeness of design problem specification is only with respect to design problem solution specification and the design principle specification, which supports the formal derivation of the latter from the former. Stork's principles are in the domain of domestic energy management and Cummaford's in electronic commerce (the leisure, pleasure and education of Carroll's extensions to HCI, since 1989, which he claims the Discipline Conception cannot express). The 'complete-for-purpose' specifications, along with the additional value-based difference between actual and desired performance, that is the design problem of 'humans interacting with computers to perform work effectively' (see Wild's paper later) demonstrates that: (1) EU research is far from 'snarled in the intellectual trap of the Discipline Conception; but that the latter is a necessary pre-requisite for the Design Problem Conception; (2) The latter's analysis is far from 'nihilistic', since it is a necessary pre-requisite for HCI design principles, which show promise of offering more reliable HCI design knowledge to date, than any other types of such knowledge; and (3) The two Conceptions offer clear criteria by which their effectiveness can be judged, so meeting Carroll's requirement for social construction (without being abjectly subjectivist). Thus, both Carroll's technical issues and philosophical 'swipes' are considered to be countered.

Dix's paper (2010) focusses on the challenge of methodological thinking in HCI. He raises the issue of 'work' as the (too limited) scope of HCI engineering (see also Carroll, 2010; Wild, 2010); but notes the later expression as 'any activity seeking effective performance', so including some leisure, domestic, and entertainment activities. Dix also raises the issue of developing (more) reliable HCI design knowledge (the original motivation of the two Conceptions). In this respect, he argues the need for validation of such knowledge by justification and evaluation. I concur completely. However, I have argued elsewhere that validation needs to include: conceptualisation; operationalisation; test (Dix's evaluation); and generalisation (Long, 1996, 
1997, 1999). Further, without some kind of consensus conception (as part of Dix's 'common ground'), researchers cannot validate, or even compare, each other's work, so making design knowledge more reliable. If we cannot agree on what is a design problem (or whatever), how can we possibly agree which model/method/principle prescribes a design solution and so validate the knowledge (Long, 1997)? Hence, the need for conceptualisation and so, conceptions.

Wild's paper (2010) applies the HCI Design Problem Conception to services and services research. He raises some critical issues for EU research. First, whether HCI engineering can address social; hedonic; and experiential concerns, as required by the design of some services. Also, whether it can accommodate aesthetics, experience, emotion and value(s) (see also Carroll and by Dix earlier). If these concerns can be even partially specified, they can be represented by the Design Problem Conception (see Lambie, et al. (1998), as concerns co-operative work and Lambie and Long (2002), as concerns the engineering of CSCW). For example, a computer games 'fun' interactive (work) system would seek to transform the state of the games playing user in terms of a domain object 'experience' (from 'undesired to desired'), made up of two sub-objects 'fun' (from 'none to much') and 'emotion' (from 'none to good'). The user, as part of the worksystem might accrue motivational ('conative') and emotional ('affective'), as well as 'cognitive' costs. However, HCI Engineering Principles could only be developed more widely (another of Wild's issues), if the associated design problems were 'hard' (see Carroll earlier). In the absence of such problems, principles could not be developed more widely. Wild rightly concludes, that 'either we need to consider in more depth what it would mean to 'engineer 'such services, including experience or we need to work out the relationship between the different perspectives on development'. My view is that both approaches should be pursued and related (see my 'Hopes' for HCI later). Last, Wild raises the issue of 'value(s)' (value; quality; choice; worth etc.), as critical for service design and how they might relate to service effectiveness. The Conceptions express a design problem, as the difference between actual and desired performance for some worksystem with respect to its domain of application, specified as how well the work is performed ('task quality') and the workload ('user costs') in performing the work that well. Value would be expressed as (part of) the rationale for (re)designing the system, such that actual equals desired performance (see also Hill, 2010). Alternatively, if value is to be part of a value (work) system, then it should be treated as 'fun' earlier, for example, by postulating a 'value' domain object (transformed from 'nil to positive'). Eitherway, the Conceptions can accommodate value(s) and relate them to effectiveness.

Hill's paper (2010) reports research, using both Conceptions to develop models of the UK Emergency Management Response System. The latter co-ordinates the emergency services, including police, medical and fire, when they respond to disasters. A method is proposed, which uses the models to diagnose design problems and to support reasoning about design solutions. 
Together, the models and method constitute design knowledge. This research raises two critical issues for EU research.

First, the relationship between design problems and user requirements (Denley and Long, 2001). Hill never mentions the latter, generally considered to be the starting point for the HCI practice of system development. Support for such development surely requires some view of how user requirements and design problems might relate. Following the two Conceptions, design problems occur, when actual performance does not equal (usually less than) desired performance (see Wild earlier). In contrast, user requirements have no such constraints. I suggest, then, a non-coextensive relationship. All design problems can be expressed as (potential) user requirements; but not vice versa. This difference needs to be acknowledged both by HCI research and practice (see Salter later and this issue, who addresses the same problem, in terms of 'client requirements').

The second critical issue, raised by Hill, is the relationship between 'models and methods' research and Principles research (see Stork, 1999; Cummaford, 2007 earlier). Hill recognises 'validated design principles, supporting general solutions to general classes of design problem, as the most effective support for practice in the longer term'; but does not identify a relationship between the two types of research. Whether, for example, one can build on the other and in particular, whether her work can, in some way, form the basis of Principles research. It may be that there is no relation of this kind. However, since both types of research share the same Conceptions, this seems unlikely. The relationship may have been implicit or poorly understood (or both), when Hill began the research (who were the supervisors, I wonder?). The most plausible set of relations, I would suggest, are as follows. 'Models and methods' research shows promise to be carried forward into Principles research, if it succeeds in specifying the models and methods themselves, in terms of the Design Problem Conception (as in Hill's research). It is more promising, if the models and methods also support the diagnosis of design problems (again, as in Hill's research). It is even more promising, if the models and methods prescribe design solutions to the diagnosed design problems (only informally and by way of illustration in Hill's research). However, it is most promising, if the problems and solutions are completely specified, for Principles research to attempt to identify the commonalities (and the non-commonalities) between them, to support the formulation of a principle by which the solution is formally derivable (or not) from the problem. These are all ways in which 'methods and models' research can support Principles research.

Salter's paper (2010) applies the HCI Discipline Conception to economic systems. He does so by means of a generic conception of an engineering discipline. This raises two important issues for EU research. The first is the scope of HCI and the expression of its general design problem. Long and Dowell (1989) assumed these to be the same for all of the HCI disciplines of craft, applied 
science and engineering (which, in contrast, differed in their knowledge and practices). Salter's generic conception sets out criteria by which a discipline can be considered an engineering one. For example, 'Criterion 1: The description of the general problem should describe the requirements component and the artefact component of the problem and the relationship between them'. Long and Dowell did this for HCI engineering, expressing its problem as: 'to design users interacting with computers to perform work effectively', such that actual equals desired performance. However, because Salter's criterion is specific to engineering, it leaves open the possibility that other HCI disciplines may have a different expression. For example, craft and applied science disciplines rarely, if ever, refer to the domain. In contrast, the domain is critical to both engineering Conceptions, as it grounds the worksystem and is the basis for 'task quality' and so effectiveness, along with 'user costs'. Possible differences between HCI disciplines expression of its general problem would have implications for the relations between disciplines and so consensus (and discipline progress).

The second issue, raised by Salter, concerns the distinction he makes between empirical and formal techniques, for example, as they appear in his Design Practice Exemplars (Fig. 8) and his Research Exemplars (Fig. 9). Neither Conceptions address this point generally, except in the case of the formality of design principles. However, there appears to be a need to relate user requirements to design problems, in the case that the former are insufficiently specified to qualify as the latter (see Hill's paper earlier). Hence, the relation cannot be formal, unlike a principle's derivation of a solution from a problem; but only 'informal' (preferred to Salter's 'empirical'). The distinction, then, between formal and informal knowledge (Salter's techniques) needs to be at least more generally referenced by the Conceptions.

I would like to bring these reflections to a close by celebrating the future of HCI in the form of some 'Fond Hopes'. First, I hope there will be more festschrifts, perhaps even of the more traditional kind, celebrating individual legacies, with more confidence, born of an increased consensus of what HCI is, what it does and how well it does it.

Second, I hope that HCI research improves the effectiveness of the design knowledge, which it acquires to support HCI design practices (a hope shared by festschrift authors - knowledge which is 'more assured' (Carroll), 'more reliable' (Dix) and 'offering a better guarantee' (Hill). Anyone who doubts this need should seriously consider: (1) How much interface design is performed by IT professionals outside the HCI community; (2) How little actual design, as opposed to related studies or evaluation, is carried out by individual HCI practitioners (as consultants) or even by those working as teams in large organisations; and (3) How much design is performed with little or no reference to HCI design knowledge (of any or no conception), other than perhaps evaluation. But how is this much needed improvement in HCI design knowledge to be achieved? In my view, It can only come about, if HCI research and practice diagnose more 
design problems and prescribe more design solutions and in so doing evaluate the effectiveness of HCI design knowledge (of whatever kind).

Third, I hope that HCI engineering (both as validated design principles and as models and methods as their pre-cursor - see Hill earlier) continues to be pursued, as one among a number of alternative and equally legitimate approaches to HCI. HCI needs all the help it can get. Inventors will be needed to invent in ways likely to be neither understood nor codifiable. Craft will be needed to address 'soft' problems and, along with other approaches, to provide an initial response to revolutionary technological and socio-cultural changes. Applied science will be needed by those either wishing to understand HCI better or who wish to borrow and transform psychology/sociology/anthropology, ethnomethodology, etc. models and methods into HCI ones. But I hope HCI engineering will also be needed to codify the design knowledge required to diagnose design problems and to prescribe design solutions for 'hard' problems. I wish it well.

\section{References}

Carroll, J.M., 2010. Conceptualizing a possible discipline of human-computer interaction. Interacting with Computers 22 (1), 3-12.

Cummaford, S.J.O., 2007. HCI Engineering Design Principles: Acquisition of ClassLevel Knowledge. Unpublished Doctoral Thesis, University College London.

Denley, I., Long, J.B., 1997. A planning aid for human factors evaluation practice. Behaviour and Information Technology 16 (4/5), 203-219.

Denley, I., Long, J.B., 2001. Multidisciplinary practice in requirements engineering: problems and criteria for support. In: Blandford, A., Vanderdonckt, J., Gray, P. (Eds.), People and Computers XV - Interaction without Frontiers. Joint Proceedings of HCI 2001 and IHM 2001. Springer Verlag, London.

Dix, A., 2010. Human-computer interaction: a stable discipline, a nascent science, and the growth of the long tail. Interacting with Computers 22 (1), 13-27.

Dowell, J., Long, J.B., 1989. Towards a conception for an engineering discipline of human factors. Ergonomics 32, 1513-1535.

Dowell, J., Long, J., 1998. Target paper: conception of the cognitive engineering design problem. Ergonomics 41 (2), 126-139. 
Hill, B., 2010. Diagnosing co-ordination problems in emergency management response to disasters. Interacting with Computers 22 (1), 43-55.

Hill, B., and Long, J., 1996. A preliminary model of the planning and control of the combined response to disaster. In: Proceedings of the 8th European Conference On Cognitive Ergonomics (ECCE8), Granada, Spain, pp. 57-62.

Lambie, T., Long, J., 2002. Engineering CSCW. In: Blay-Fornarino, M., Pinna-Dery, A.M., Schmidt, K., Zarate, P. (Eds.), Co-operative Systems Design: A Challenge of the Mobility Age. IOS Press, Amsterdam.

Lambie, T., Stork, A., and Long, J., 1998. The co-ordination mechanism and cooperative work. In: Proceedings of the 9th European Conference On Cognitive Ergonomics (ECCE9), Limerick, Ireland, pp. 163-166.

Lim, K.Y., Long, J.B., 1994. The MUSE Method for US Ability Engineering. Cambridge University Press, UK.

Long, J., 1980. Effects of prior context on two-choice absolute judgements without feedback. In: Nickerson, R.S. (Ed.), Attention and Performance VIII. Erlbaum, Hillsdale, NJ.

Long, J., 1995. Commemorating Donald Broadbent's contribution to the field of applied cognitive psychology: a discussion of the special issue papers. Applied Cognitive Psychology 9 (S1), 197215 .

Long, J., 1996. Specifying relations between research and the design of human- computer interactions. International Journal of Human-Computer Studies 44 (6), 875-920.

Long, J., 1997. Research and the design of human-computer interactions or "whatever happened to validation'? In: Proceedings of HCI'97, Bristol, pp. 223-243.

Long, J., 1999. Specifying relations between research and the practice of solving applied problems: an illustration from the planning and control of multiple task work in medical reception. In: Gopher, D., Koriat, A. (Eds.), Attention and Performance XVII. MIT Press, Cambridge, MA, pp. 259-284.

Long, J.B., Dowell, J., 1989. Conceptions for the discipline of HCI: craft, applied science and engineering. In: Sutcliffe, A., Macaulay, L. (Eds.), Proceedings of the Fifth Conference of BCS HCI SIG. Cambridge University Press, UK. 
Long, J.B., Whitefield, A.D. (Eds.), 1989. Cognitive Ergonomics and Human- Computer Interaction. Cambridge University Press, UK.

Salter, I.K., 2010. Applying the conception of HCI engineering to the design of economic systems. Interacting with Computers 22 (1), 56-57.

Smith, M.W., Hill, B., Long, J.B., Whitefield, A.D., 1997. Modelling the relationship between planning, control, perception and execution behaviours in interactive worksystems. In: Monk, York A., Diaper, D., Harrison, M. (Eds.), Proceedings of the Seventh BCS HCI SIG Conference. Cambridge University Press, UK.

Stork, A., 1999. Towards Engineering Principles for Human-Computer Interaction (Domestic Energy Planning and Control). Unpublished Doctoral Thesis, University College London.

Timmer, P., Long, J., 2002. Expressing the effectiveness of planning horizons. Le Travail Humain $65(2), 103-126$.

Wikipedia, 2009. The free encyclopaedia - see under 'Festschrift'.

Wild, P.J., 2010. Longing for service: bringing the UCL conception towards services research. Interacting with Computers 22 (1), 28-42.

\section{Further reading}

Long, J., 2010. Some celebratory HCI reflections on a celebratory HCI festschrift. Interacting with Computers 22 (1), 68-71. 